\title{
Localization of the Acoustic Sources of the A340 with a Large Phased Microphone Array During Flight Tests
}

\author{
J. F. Piet * \\ ONERA, Office National d'Études et de Recherches Aérospatiales, Toulouse, France \\ $\mathrm{U}$. Michel ${ }^{\dagger}$ and P. Böhning ${ }^{\ddagger}$ \\ DLR, Deutsches Zentrum für Luft- und Raumfahrt, Berlin, Germany
}

\begin{abstract}
$\underline{\text { Abstract }}$
Flyover measurements with a phased array of microphones extending over an area of $16 \mathrm{~m}$ by $16 \mathrm{~m}$ are reported. The 161 microphone array was made possible by combining hardware from ONERA and DLR. In this investigation of the airframe noise of an Airbus A340, the flyover altitudes were between $90 \mathrm{~m}$ and $165 \mathrm{~m}$. The data reduction methods for moving objects of DLR and ONERA are compared. Some source maps are shown and discussed. It is demonstrated that nested arrays must be used for a study over a wide frequency range, and that comparisons of the noise maps between different arrays provide valuable information about the noise sources. The ONERA method is shown to be a powerful data reduction method based on a small number of microphones while the DLR method results in alias-free maps at the expense of a much larger number of microphones.
\end{abstract}

\section{Introduction}

It is known from flyover noise measurements with single microphones that airframe noise is a major contributor to the noise emission of modern aircraft during their landing approach. The directivity of the aircraft overall noise source might be derived from this measurement. However, the identification and the contribution of each individual source and its directivity can only be derived from flyover tests with the help of phased arrays of microphones. DLR and ONERA have both applied this technique to landing aircraft (Michel et al., ${ }^{1}$ Piet et al., ${ }^{2}$ Michel and $\mathrm{Qiao}^{3}$ ).

A flyover test campaign was performed with an Airbus A340, in the framework of the European Research Program RAIN (Chow et al., ${ }^{4}$ Davy et al. ${ }^{5}$ ), to investigate the airframe noise sources for different aircraft settings, from the aircraft in the cruise configuration ("clean" aircraft) to the complete landing configuration with all high-lift devices fully deployed and landing gear down. To reduce engine noise as much as possible, the engines were operated at ground idle setting. Flyover altitudes in the order of $100 \mathrm{~m}$ to $150 \mathrm{~m}$ were necessary for safety reasons and required a much larger array than ever used before by either of the part-

\footnotetext{
* jean-francois.piet@onecert.fr, phone : +33 5622525 25, fax : +33562 252583 .

†ulf.michel@dlr.de, phone : +49 30 310006-26, fax : -39

‡peer.boehning@dlr.de, phone : +49 30 310006-48, fax : -39 Copyright (c) 2002 by ONERA and DLR. Published by the American InCopyright () 2002 by ONERA and DLR. Published by
stitute of Aeronautics and Astronautics, Inc. with permission.
}

ners. By combining the hardware available at ONERA and DLR it was possible to build a microphone array of $16 \mathrm{~m}$ by $16 \mathrm{~m}$ consisting of 161 microphones.

The principle is first recalled and the methods presented. Then, flight tests are introduced and typical results are discussed in terms of array performance.

\section{Source localization on a flying aircraft}

\section{$\underline{\text { De-Dopplerized microphone signals }}$}

The frequency $f_{e}$ emitted by a tonal sound source on an aircraft flying with a flight Mach number $M$ is related to the frequency $f$ observed on the ground by

$$
f=f_{e} D_{f}
$$

with the definition of the Doppler factor,

$$
D_{f}=1 /\left(1-M \cos \theta_{e}\right),
$$

where $\theta_{e}$ is the emission angle relative to the flight direction.

For a flight Mach number of $M=0.25$, a tone on the aircraft is heard on the ground with a frequency of up to $1.33 f$ when the aircraft approaches and as low as $0.8 f$ after the aircraft has passed by. This Doppler frequency shift complicates the investigation of tones and frequency spectra emitted by the aircraft with microphones on the ground. Another problem for the analysis of the noise during an aircraft flyover is the amplitude change due to the variable distance of the 
aircraft from the microphones. By assuming a single monopole source and an ideal propagation, both effects can be eliminated by a de-Dopplerization and normalization of the microphone signals according to

$$
p_{d}\left(t_{e}\right)=p\left(t_{e}+\tau\left(t_{e}\right)\right) \tau\left(t_{e}\right) / \tau_{r e f},
$$

where $t_{e}$ is the emission time of the sound wave at the source position which must be known as a function of time $t_{e}$, and $p\left(t_{e}+\tau\right)$ is the signal of the microphone at the reception time $t=t_{e}+\tau\left(t_{e}\right)$. The propagation time $\tau\left(t_{e}\right)$ of the waves can be determined in quiescent air with constant sound speed $c$ by

$$
\tau\left(t_{e}\right)=r\left(t_{e}\right) / c
$$

where $r\left(t_{e}\right)$ is the distance between the known moving source position and the microphone on the ground at the time of emission.

The expression $\tau / \tau_{\text {ref }}$ in Eq. (3) is equal to the ratio of the actual distance $r$ to a reference distance $r_{r e f}$ in the case of no wind. The time lag $\tau\left(t_{e}\right)$ removes the frequency shift while the distance ratio $r / r_{\text {ref }}$ removes the influence of the spherical attenuation of the sound pressure due to the variable source-microphone distance during an aircraft flyover.

A time series for the de-Dopplerized signal $p_{d}\left(t_{e}\right)$ has to be determined by re-sampling the measured time series $p(t)$ at the necessary time steps. The re-sampling frequency $f_{s, e}$ of the de-Dopplerized time series $p_{d}\left(t_{e}\right)$ may be different from the original sampling frequency $f_{s}$ of the microphone signal $p(t)$. However, a reduction of this frequency is limited by the requirements of the sampling theorem.

\section{Interpolation errors}

If the re-sampling is done by a linear interpolation between the available samples, the original signal is replaced by a polygon. The amplitude loss of a sine wave depends on the ratio

$$
f / f_{s}=\left(f_{e} / f_{s}\right) D_{f}
$$

where $f_{s}$ is the sampling frequency of the original time series. The highest possible amplitude losses are described by

$$
\Delta \mathrm{SPL}_{\max }=20 \lg \left(\cos \left(\pi\left(f / f_{s}\right)\right)\right)(\text { in } \mathrm{dB}) .
$$

The average loss is assumed to be half this value. Typical values are tabulated in Table 1 . It can be seen that for a sampling frequency $f_{s}=33.3 \mathrm{kHz}$ of the original time series, the error is negligible only for frequencies $f<2 \mathrm{kHz}$ which means that the sampling

\begin{tabular}{|c|c|c|c|}
\hline$f / f_{s}$ & \multicolumn{2}{|c|}{ frequency $f$ of interest } & \multirow{2}{*}{$\begin{array}{c}\text { SPL } \\
\text { aB }\end{array}$} \\
\cline { 2 - 3 } & $f_{s}=33.3 \mathrm{kHz}$ & $f_{s}=20 \mathrm{kHz}$ & -3.0 \\
\hline $1 / 3$ & $11.1 \mathrm{kHz}$ & $6.7 \mathrm{kHz}$ & -1.5 \\
\hline $1 / 4$ & $8.3 \mathrm{kHz}$ & $5.0 \mathrm{kHz}$ & -0.9 \\
\hline $1 / 5$ & $6.7 \mathrm{kHz}$ & $4.0 \mathrm{kHz}$ & -0.6 \\
\hline $1 / 6$ & $5.6 \mathrm{kHz}$ & $3.3 \mathrm{kHz}$ & -0.6 \\
\hline $1 / 7$ & $4.8 \mathrm{kHz}$ & $2.9 \mathrm{kHz}$ & -0.5 \\
\hline $1 / 8$ & $4.2 \mathrm{kHz}$ & $2.5 \mathrm{kHz}$ & -0.3 \\
\hline $1 / 10$ & $3.3 \mathrm{kHz}$ & $2.0 \mathrm{kHz}$ & -0.2 \\
\hline $1 / 16$ & $2.1 \mathrm{kHz}$ & $1.3 \mathrm{kHz}$ & -0.1 \\
\hline
\end{tabular}

Table 1 Average amplitude loss $\triangle \mathbf{S P L}_{a v}$ due to re-sampling by a linear interpolation.

frequency should be 16 times the frequency of interest if linear interpolation is used. This error can be reduced by improving the interpolation method.

Due to the influence of the emission angle on the frequency ratio $f / f_{s}$ according to Eq. (5), the attenuation will be larger in the forward arc than in the rear arc.

The polygon approximation also increases the noise level of the interpolated signal as was shown by Howell et al. ${ }^{6}$ They conclude that a factor $f / f_{s}=1 / 4$ must be satisfied.

\section{Focused sound pressure of an acoustic antenna}

The localization of sound sources in high-speed motion requires the use of an acoustic antenna which consists of an array of microphones and an appropriate signal-processing software. It is indeed the only localization tool that can accommodate such short observation times.

The array consists of $N$ microphones at the positions $\left(x_{i}, y_{i}, z_{i}\right), i=1, N$ and yields $N$ microphone signals $p_{i}(t)$. These are digitized (at the time of reception) and stored for later data processing. The sound pressure of the acoustic antenna (the localization function), when focused at a position $\left(x_{e}\left(t_{e}\right), y_{e}\left(t_{e}\right), z_{e}\left(t_{e}\right)\right)$, is given by the sum of the de-Dopplerized signals of all $N$ microphones in the array.

$$
p_{f}\left(t_{e}\right)=\frac{\sum_{i=1}^{N} p_{i}\left(t_{e}+\tau_{e i}\right) g_{i} \tau_{e i} / \tau_{r e f}}{\sum_{i=1}^{N} g_{i}}
$$

where $p_{i}\left(t_{e}+\tau_{e i}\right)$ is the signal of microphone $i$ at the time of reception $t=t_{e}+\tau_{e i}$ and $g_{i}$ is a weighting (spatial shading) factor of the microphone signal in the data analysis. The delays $\tau_{e i}\left(t_{e}\right)$ must be precisely known for any possible source location on the moving object and any emission time $t_{e}$. In the case of a flying aircraft, it has to be deduced from the available tracking of the aircraft using interpolations (both spatial 
and temporal). The quality of the results depends on the accuracy of the de-Dopplerization.

For the case of a single monopole source in the assumed focal position, the result of Eq. (7) is equal to the sound pressure of a single microphone in the reference distance $r_{r e f}=\tau_{r e f} / c$.

Focused narrow-band frequency spectra in the time domain

Generally one is not interested in the sound pressure according to Eq. (7) but in the averaged meansquare sound pressure or its power spectrum $W_{p p}\left(f_{e}\right)$. This can be determined with Fast Fourier Transforms (FFT) from the focused pressure $p_{f}\left(t_{e}\right)$ in Eq. (7),

$$
W_{p p}\left(f_{e}\right)=\frac{1}{M} \sum_{m=1}^{M} P_{m} \times P_{m}^{*}
$$

where $P_{m}$ is the discrete complex frequency spectrum of the focused sound pressure $p_{f}\left(t_{e}\right)$ for a time segment $m$ and $P_{m}^{*}$ is its conjugate complex. The averaged power spectrum is computed from $M$ overlapped time segments distributed over the averaging time $T$.

Despite the de-Dopplerization and distance normalization in Eqs. (3) and (7), the sound-pressure signal is not stationary random because the emission angle changes with time during a flyover. Therefore, the averaging time $T$ has to be kept rather short, which limits the statistical stability of the results.

\section{Beam pattern, side lobes and aliases}

In the case of a single moving monopole source, the localization function (Eq. (8)) reaches its maximum value when the focus is located at the position of the source. If the sound-pressure level is plotted as a function of the distance between focal position and actual source position we obtain the beam pattern of the antenna. The beam width is generally defined where the sound-pressure level has decreased by $3 \mathrm{~dB}$ in comparison to the peak level and is proportional to the ratio of the product of the wave length $\lambda$ and the distance of the source from the array over the size $s$ of the array as seen from the source.

Since the observed frequency $f$ is higher (and the wave length $\lambda$ smaller) for small emission angles $\theta_{e}$ (forward arc) than for large angles (see Eq. (1)), the beam width is smaller in the forward arc than in the rear arc of a flying aircraft. This dependence on emission angle is illustrated in Fig. 1 with a simulation of a moving sinusoidal point source. The array consists of 41 microphones in a cross layout, equal to the $8 \mathrm{~m}$ by $8 \mathrm{~m}$ array of ONERA.
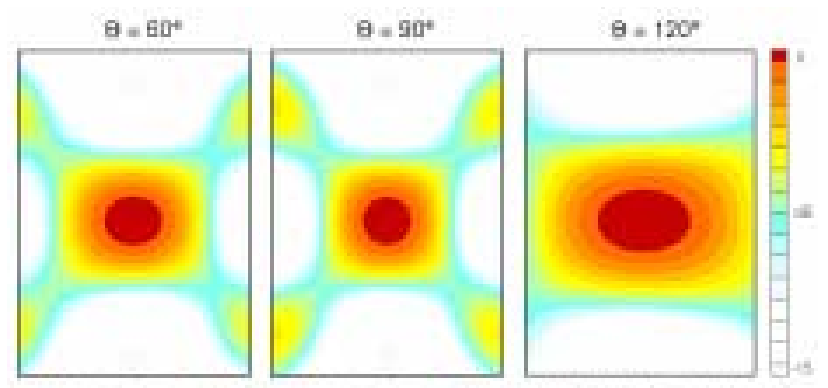

Fig. 1 Influence of emission angle on the beam width for a tone of $f=1000 \mathrm{~Hz}$ moving with a flight speed of $80 \mathrm{~m} / \mathrm{s}$ at an altitude of $100 \mathrm{~m}$.

The focused sound-pressure level will not decrease continuously with increasing distance between focus and source but will exhibit secondary peaks, called side lobes and aliases. Side-lobes are a result of the limited size of the microphone array and their pattern depends on the microphone arrangements and the choice of the weighting factors. Side lobes can be seen in the beam patterns for 60 and $90^{\circ}$ in Fig. 1.

Spatial aliases are a consequence of the insufficient spatial discretization of the acoustic field by the array. Aliases limit the upper frequency that can be resolved with regularly spaced microphone arrays. The maximum usable frequency of an array for an aircraft flyover is defined by

$$
f_{\max }=\frac{z_{e} c}{l \Delta x}
$$

where $z_{e}$ is the flyover altitude, $\Delta x$ the microphone separation and $l$ the aircraft size. Some results are shown in Table 2.

\begin{tabular}{|c|c|c|}
\hline $\begin{array}{c}z_{e} \\
(\mathrm{~m})\end{array}$ & $\begin{array}{c}\Delta x \\
(\mathrm{~m})\end{array}$ & $\begin{array}{c}f_{\max } \\
(\mathrm{Hz})\end{array}$ \\
\hline 120 & 0.8 & 850 \\
\hline 120 & 0.4 & 1700 \\
\hline 120 & 0.2 & 3400 \\
\hline 100 & 0.8 & 708 \\
\hline 100 & 0.4 & 1417 \\
\hline 100 & 0.2 & 2833 \\
\hline
\end{tabular}

Table 2 Maximum frequency $f_{\max }$ (Eq. (9)) of a line array as function of source distance $z_{e}$ and microphone spacing $\Delta x$ (aircraft size $l=60 \mathrm{~m}$ ).

\section{The localization methods of ONERA and DLR}

ONERA uses a method described by Elias $^{7}$ for a reduction of the side lobes in cross arrays consisting of two line arrays. The procedure results in a good two-dimensional localization with a small number of 
microphones. ${ }^{8}$ Nested arrays are required to cover a large frequency range.

DLR uses a planar array with non-regular microphone locations which reduce the level of aliases substantially at the expense of a larger microphone number. Eq. (8) is used for the analysis. Experience with these arrays has shown that a non-regular microphone distribution does not eliminate the need of using smaller arrays for higher frequencies. Therefore, nested arrays are required independently of the used microphone layout.

\section{Localization maps and interpretation}

\section{Presentation of results in the form of maps}

The source distribution on the aircraft can be studied with the help of color coded maps created by calculating the focused frequency spectra on a grid including the possible positions (supposed to fit in a plane) of the sources on the aircraft. The array depthof-focus (ability to discriminate sources depth-wise) is poor, so sources will (fortunately) be detected even if they are not exactly in the focus plane. Because of the frequency dependence of the beam width, the maps have to be calculated separately for each frequency in narrow bands which may then be added to one-third octave bands.

\section{Array intrinsic properties}

The interpretation of localization maps in the presence of extended sources is a matter of discussion even in static cases $\left(\operatorname{Sen}^{9}\right)$. The focused beam-forming method limitations are briefly recalled here.

This method assumes that the source is a point monopole while real sources might be extended, partly or totally correlated, and directive.

As mentioned earlier, in the ideal case of a single monopolar source, the array pattern has a main beam (the width of which mainly depends on the ratio of array size over the distance of the sources and on the angle of observation) and side lobes.

The position of the localization estimator maximum on the map indicates the true source position, unless the source is not in the focus plane. In that case, a parallax error is possible. The estimator maximum value reflects the source amplitude, in the case of an ideal propagation.

In all cases, the map is a convolution of the beam pattern with the source distribution. In the situation of several point or line sources, the indicated source amplitude in each position is influenced by theoretically all other sources. Only for point sources sufficiently distant from one another (with respect to the beam-width), we can conclude that the position of the maximum is almost correct and that the indicated strength is independent of the beam-width and correct.

For real sources, the maps show several source areas, more or less extended, with multiple maxima, depending on both the beam pattern and the spatial extend of the source. The beam-forming method is robust, i.e., even in these cases, the maps give source positions and a source ranking, provided the beam width is small enough. However, a simple interpretation of a source located at each maximum and with an identified amplitude is not possible any more : levels are not absolute and especially the map cannot be interpreted as an acoustic density map. The source position may be biased in the case of non-monopole sources. This may be especially true for spinning modes from the engine inlet or the nozzle.

Alternative solutions compared to beam-forming were proposed in static cases, either by deconvolution of the beam pattern on the results or by the use of a multipole source model (Mosher, ${ }^{10}$ Varnier et al. ${ }^{11}$ ). These methods have not been applied to flight tests yet, because of the motion and Doppler effect induced,

\section{Effect of aircraft motion}

With the aircraft high-speed motion, the situation is further complicated by the Doppler-shifted frequency (see Fig. 1) and by the fast change in the beam-width of the localization function with the geometric parameters (distance and angle between focus point and microphone).

Source directivity effects can be studied by computing the maps for different emission angles. The array performance is best when the sources are vertically above the array $\left(90^{\circ}\right)$ while map calculations for higher or lower angle values suffer from a deterioration of array performance (beam width, parallax effects...).

Moreover, new problems have to be dealt with, regarding the experimental situation, especially the propagation media and the statistical analysis of the results because of the short observation times. This will be briefly discussed in the last section.

\section{Application to flight tests}

The Airbus A340 test aircraft performed specific flights dedicated to airframe noise measurement on September $29^{t h}$ and October $9^{t h}$, 1998. It flew above the DLR-ONERA array of microphones with several combinations of different slat-and-flap and landinggear settings, in an airspeed-range typical of the usual 
landing-approach situation. The test site was located on the extended centerline of the runway of Tarbes airport. This allowed the pilots to fly above the array at altitudes between 90 and 165 meters depending on the safety margin required.

\section{Definition of the microphone arrays}

A total of 161 condenser measuring microphones were installed (1/4 inch model). ONERA's 81 microphones were distributed in three cross-shaped subarrays of homothetic sizes of 16,8 and 4 meters and microphone spacings of $0.8,0.4$ and 0.2 meters, respectively. The largest sub-array was devoted to the lowest frequencies and therefore named "Very Low Frequency" (VLF) array, and the other two, respectively, "Low Frequency" and "High Frequency" arrays.

DLR used an array consisting of the 41 microphones of the smallest ONERA array and 80 additional nonregularly positioned microphones, which were concentrated near the center yielding an effectively slightly smaller size than the smallest ONERA array. It is called the Very High Frequency (VHF) array.

The four sub-arrays altogether cover the frequency range from $400 \mathrm{~Hz}$ up to $8 \mathrm{kHz}$ with a spatial resolution enabling the separation of the expected main sources from one another. An investigation for frequencies lower than $400 \mathrm{~Hz}$ would require arrays of an even greater size. The analysis of the highest one-third octave band of $10 \mathrm{kHz}$ is of minor importance because the sound-pressure level is reduced considerably by atmospheric absorption.

The spatial resolution of the chosen arrays is shown in Table 3 for a flyover altitude of $120 \mathrm{~m}$ and an emission angle of $90^{\circ}$. The very-low frequency array (size $16 \mathrm{~m}$ ) is well suited for the $800-1600 \mathrm{~Hz}$ range with a resolution between $1.0 \mathrm{~m}$ and $1.9 \mathrm{~m}$. The lowfrequency array (size $8 \mathrm{~m}$ ) has the same performance between $1600 \mathrm{~Hz}$ and $3150 \mathrm{~Hz}$, the high-frequency array (size $4 \mathrm{~m}$ ) from $3150 \mathrm{~Hz}$ to $6300 \mathrm{~Hz}$, and the very-high frequency array from $4000 \mathrm{~Hz}$ to $8000 \mathrm{~Hz}$.

The array is shown in Fig. 2. The microphones were installed on wooden plates. The space between the edges of the plates and the ground was filled with sand to reduce acoustic scattering.

The microphones were mounted with their axes oriented perpendicularly to the flight-path at grazing incidence. Their membranes were assumed to be located in the reflection plane resulting in a doubling of the sound pressure. However, this requirement is not satisfied for very high frequencies, resulting in underestimated sound-pressure levels.

The locations of nine microphones were measured by

\begin{tabular}{|c|c|c|}
\hline $\begin{array}{c}f \\
(\mathrm{~Hz})\end{array}$ & $\begin{array}{c}s \\
(\mathrm{~m})\end{array}$ & $\begin{array}{c}b \\
(\mathrm{~m})\end{array}$ \\
\hline 200 & 16 & 7.7 \\
\hline 400 & 16 & 3.8 \\
\hline 800 & 16 & 1.9 \\
\hline 1600 & 16 & 1.0 \\
\hline 1600 & 8 & 1.9 \\
\hline 3150 & 8 & 1.0 \\
\hline 3150 & 4 & 1.9 \\
\hline 6300 & 4 & 1.0 \\
\hline 4000 & 3 & 1.9 \\
\hline 8000 & 3 & 1.0 \\
\hline
\end{tabular}

Table 3 Beam width $b$ of a line array for various frequencies $f$ and array sizes $s$ for an altitude $z_{e}=$ $120 \mathrm{~m}$ of the focus and an emission angle of $\theta=90^{\circ}$.

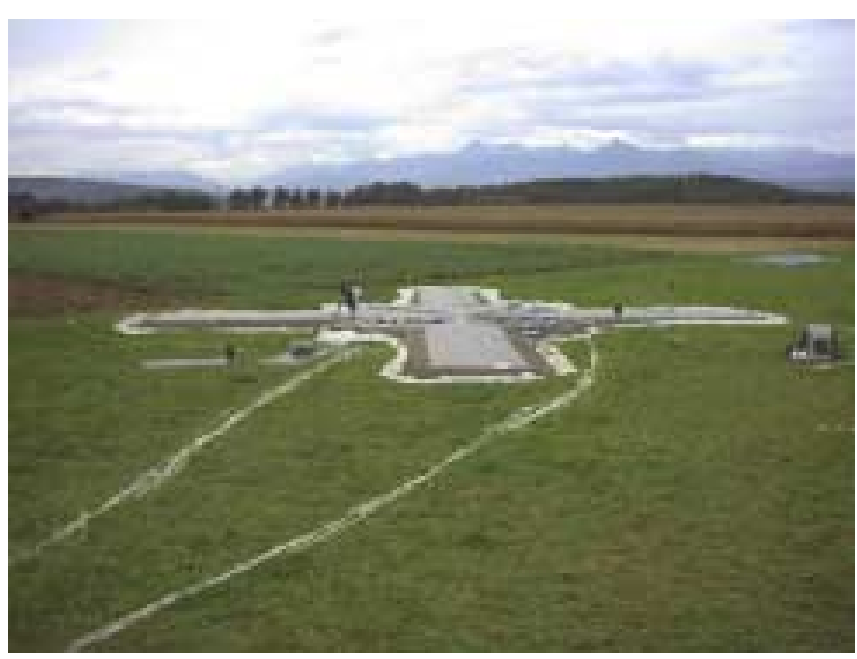

Fig. 2 Array of microphones. Flight-path is from left foreground to right background.

Airbus France using static D-GPS, with a relative accuracy estimated to $1 \mathrm{~cm}$. The position of the wooden plates was accurately adjusted so that the locations of the other microphones were deduced from the first nine.

\section{Data acquisition and reduction}

\section{Data acquisition}

The microphone signals were amplified between 10 and $40 \mathrm{~dB}$. These signals and the IRIG code derived from the GPS time (for the later synchronization with the tracking) were digitized at $33.333 \mathrm{kHz}$ with a 16-bit resolution using a DLR acquisition system with a total of 192 analog input channels. Total sampling duration was 10 seconds resulting in a file size of $122 \mathrm{MB}$ for each flyover. 


\section{Aircraft flight-path information}

Airbus France implemented the aircraft D-GPS tracking and provided after the tests the tracking of four points on the aircraft as a function of UTC time. The aircraft lateral deviation and altitude above the array are defined by the location of the main landing gear respectively to the center of the array.

\section{Statistical analysis of signal}

Random-type sources, supposedly stationary in the moving frame, have to be characterized statistically by using averages of the focused signal spectra. The averaging time has to be as large as possible to keep the statistical uncertainty small. However, this time is limited in an aircraft flyover which does not exceed a few seconds. In addition, the sound emission of the sources might change with the emission angle which also requires to keep the variation of the observation angle low during the averaging process.

Furthermore, there is a another risk in averaging the maps over a very wide solid angle. Indeed, most of the uncertainties (especially on the source position) mentioned later depend on the emission angle. Consequently, a point source seen with a position bias (parallax), that would have its "apparent" location varying too much compared to the beam-width over the integration angle would end-up being underestimated.

For these reasons, the maps are computed for time frames of about 0.5 seconds in which the emission angle changes between 15 and $20^{\circ}$ depending on airspeed and altitude.

These parameters, as will be detailed further, yield only 10 to 12 statistically independent blocks. This has the consequence of a high statistical uncertainty for a small bandwidth analysis (narrow-band or one-third octave bands with very low frequencies). However, for wider frequency bands, such as one-third octave bands at frequencies above $1 \mathrm{kHz}$, the statistical error is much reduced.

\section{Re-sampling for the de-Dopplerized time series}

As explained in the beginning, the signals have to be de-Dopplerized by re-sampling the original time series with a constant sampling frequency in the frame of the moving focus position.

DLR (for the very high frequency array) used a re-sampling frequency of $25600 \mathrm{~Hz}$, where it was assumed that the moving source did not emit signals above $12.8 \mathrm{kHz}$ or that these high frequencies were suf- ficiently attenuated by atmospheric absorption. FFTs were performed with time segments consisting of 1024 samples, yielding frequency spectra with a frequency step size of $25 \mathrm{~Hz}$. The total length of the time series was 12288 samples with 12 statistically independent time segments yielding an averaging time of 0.48 seconds.

The amplitude reduction due to linear interpolation is shown in Table 4 for five emission frequencies and the three emission angles $60^{\circ}, 90^{\circ}$ and $120^{\circ}$. The frequencies $f$ at reception time that determine the errors according to Table 1 are calculated for a flyover with a Mach number $M=0.25$.

\begin{tabular}{|c|c|c|c|}
\hline$f_{e}$ & \multicolumn{3}{|c|}{$\Delta \mathrm{SPL}_{a v}(\mathrm{~dB})$} \\
\cline { 2 - 4 }$(\mathrm{kHz})$ & $\theta_{e}=60^{\circ}$ & $\theta_{e}=90^{\circ}$ & $\theta_{e}=120^{\circ}$ \\
\hline 3.15 & -0.3 & -0.2 & -0.2 \\
\hline 4 & -0.4 & -0.3 & -0.2 \\
\hline 5 & -0.7 & -0.5 & -0.4 \\
\hline 6.3 & -1.1 & -0.8 & -0.6 \\
\hline 8 & -1.8 & -1.4 & -1.1 \\
\hline
\end{tabular}

Table 4 Influence of emission angle $\theta_{e}$ on the interpolation error during the de-Dopplerization for a flight Mach number of 0.25 and a sampling frequency of $33.333 \mathrm{kHz}$ (DLR data reduction).

ONERA, concerned with the lower frequencies, chose a lower re-sampling rate of $20 \mathrm{kHz}$ and a time series of 10240 samples yielding 10 statistically independent time segments of 1024 samples with an averaging time of 0.51 second. The original signal with the sampling frequency of $f_{s}=33.3 \mathrm{kHz}$ was first low-pass filtered at $20 \mathrm{kHz}$ with a digital filter. The resulting signal was then re-sampled at $20 \mathrm{kHz}$ in the reception time frame during the de-Dopplerization process. This resulted in further amplitude reductions not presented here, that disabled obtaining maps at $8 \mathrm{kHz}$ for $60^{\circ}$.

The amplitude loss due to re-sampling was not corrected in the results.

\section{Source characterization limitations}

The goal of the array analysis is to determine the locations and evaluate the "strength" hierarchy of the sound sources on the aircraft. The intrinsic array properties were introduced in the first section and apply here, leading to different performances depending on the specific flyover and the observation angle. Moreover, the aircraft motion (with the Doppler effect induced) and the propagation medium characteristics (because of the large distances involved) complicate the task.

The source locations can be determined with 
great confidence, although some bias error may occur, generally emphasized in the forward and rear arcs, especially with parallax effects.

- The wind speed shifts the apparent location of the sound sources.

- The wind and temperature gradients yield curved acoustic rays between sources and microphones and lead to source position distortions.

- The tracking of the aircraft is not perfect, firstly because the D-GPS and flight parameters may have a bias, secondly because the wings bend upwards in flight.

The situation is much more difficult concerning the source strength, because these limitations add-up to those inherent to the beam-forming in the presence of real extended sources.

- Among the propagation effects, the (i) atmospheric absorption along the propagation path and the (ii) coherence losses between microphones due to sound diffusion by turbulence or other spurious effect.

- The Doppler-shifted frequency introducing discrepancies in the atmospheric damping and in the beam-width over the observation angle.

- The spatial coherence within the source region.

- The microphones are mounted with grazing incidence rather than flush in the surface. Therefore, the membranes are not precisely located in the reflection plane.

- The data processing : linear interpolation of the signals yields an amplitude loss at high frequencies.

\section{Results and discussion}

Results presented in this article aim to illustrate array properties and the performance of the localization method. It is therefore not focused on aircraft aerodynamic noise characteristics.

The reproducibility of the de-Dopplerized spectra and the map levels for flights with identical configuration and similar flyover altitudes was generally (astonishingly) quite good.

\section{De-Dopplerized narrow-band spectra}

As a complement to the localization maps, we computed the average of the de-Dopplerized narrowband spectra of each individual sensor on the array for all flyovers and the three emission angles 60, 90 and $120^{\circ}$. The averaging over many microphones increases the statistical stability of the results. Unlike the localization estimator, there is no beam-forming and the "averaged de-Dopplerized spectra" reflects the average sound emission over the surface of the array. It provides a good indicator of the acoustic characteristics of the flyover, allows the identification of emission levels and reveals the presence of tones.

An example of de-Dopplerized narrow-band spectra for a flyover with slats and flaps extended, but landing gear retracted is shown in Fig. 3. Here, the radiation is generally broadband and the power of the few narrow-band peaks is almost negligible. Radiation to the rear $\left(120^{\circ}\right)$ is higher than into the forward arc for high frequencies despite the fact that the Doppler-amplification due to the aircraft motion is not corrected in any of the results. Spectra, which are usually given for a reference observation distance $(120 \mathrm{~m})$, are here presented with an arbitrary relative scale.

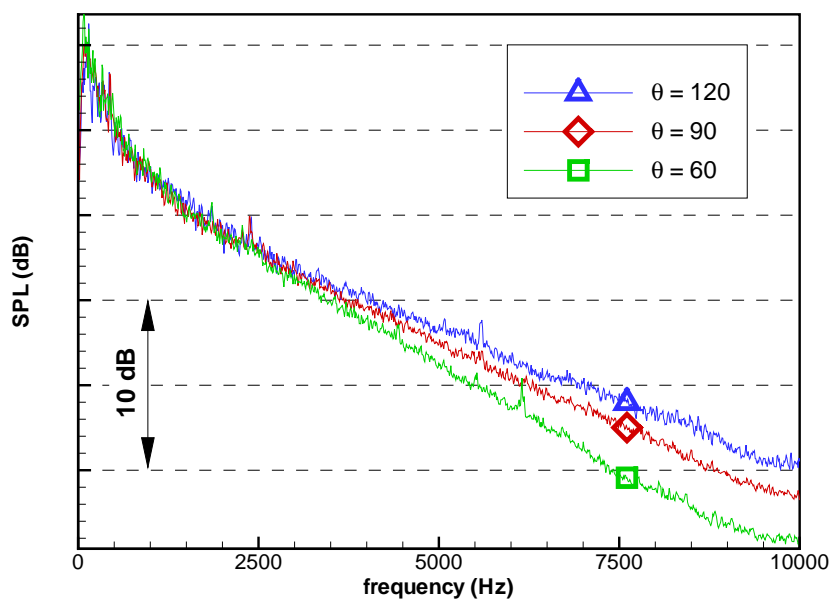

Fig. 3 De-Dopplerized narrow-band spectra of the slat-and-flap-only case for the three emission angles 60,90 and $120^{\circ}$ (relative scale).

In case the de-Dopplerized spectra contain strong peaks, related to tonal noise sources, narrow-band maps are then investigated to identify the locations of these sources.

\section{Influence of array size and geometry on maps}

The results for the campaign showed that in some cases maps could be obtained up to $10 \mathrm{kHz}$. Spatial resolution is related to main geometric parameters such as the array size and the source-array distance. Unlike for static situations, the source-array distances are generally not identical for different flyovers. This leads to different performance and induces, in the presence of extended sources, changes in their "apparent levels". Plots are presented in color with levels (in dB) 

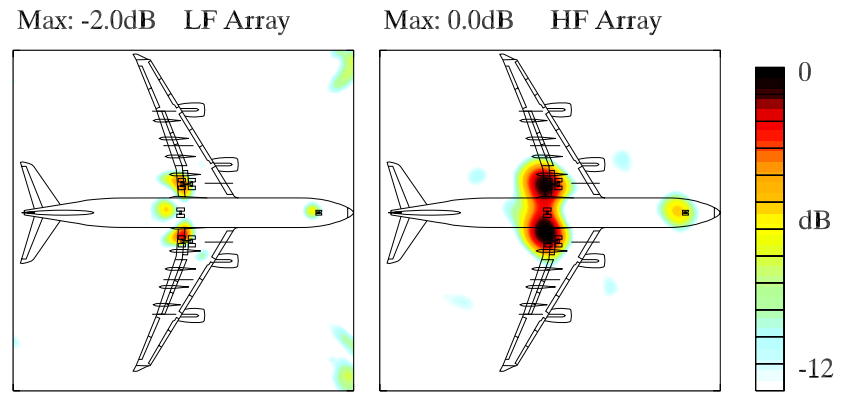

Fig. 4 Maps for a landing-gear only configuration test, in a low-frequency one-third octave band in the forward arc of emission $\left(\theta_{e}=60^{\circ}\right)$. The LF array enables the separation of the central-gear source whereas the HF array integrates the levels because of its larger beam-width.

normalized to the maximum value found on the map. In the case of a two- or three-map figure, a common scale will enable a level comparison : then, there is only one color scale bar in the right margin (Figures 4, 5, 7 and 9).

Let us take the example of a flyover achieved with landing gears down only, at an airspeed of 180 knots (see Fig. 4). The same processing is applied to LF and HF arrays, for the forward arc of emission (so both arrays "see" the same source in the same time frame $^{1}$ ). The spatial resolution doubling between both arrays leads to the ability of the larger array to separate sources from both main gears and even with the central gear. On contrary, the smaller array map is more integrated over the area. Note that the sources at the front left and front right corners for the LF array map are aliases of the main gear sources (the arms of the array are oriented at $45^{\circ}$ with respect to the fuselage). The shift of the found source position, downstream the landing gear location is due to the resulting parallax because the source is observed in its forward arc of emission.

Furthermore, the larger value in the source "apparent" level for the smaller array (with the larger beam width) indicates the situation of distributed sources. Theoretically, a linear distribution of sources with constant strength would yield an increase of $3 \mathrm{~dB}$ for the smaller array, a similar planar distribution of sources would yield an increase of $6 \mathrm{~dB}$. This is only true when the source extension is much larger than the array spatial resolution. In our case, it can be concluded that main-gear sources do not appear as point sources to the larger array. A way to get a rough estimate of the

\footnotetext{
${ }^{1}$ There is, though, a slight difference in the solid angle of observation, that might affect the maps in the case of very directive phenomena.
}
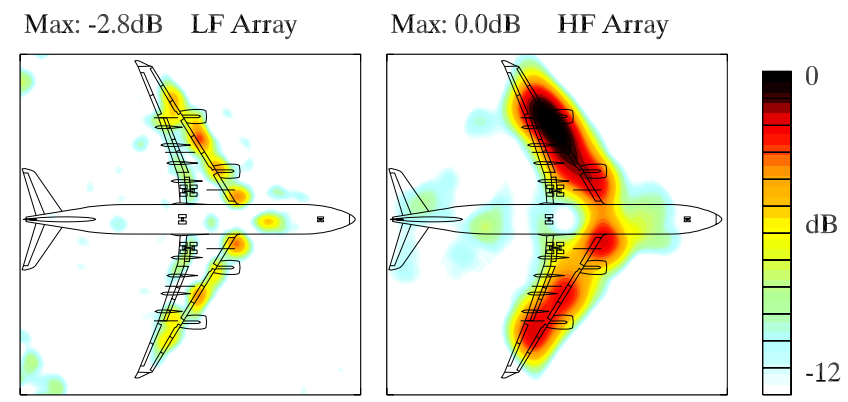

Fig. 5 Maps for a low frequency $\left(\theta_{e}=90^{\circ}\right)$ for a slat-and-flap extended configuration ; Comparison of the LF (left) and HF (right) arrays. The LF array discriminates the slat sources from those downstream the outboard left engine.
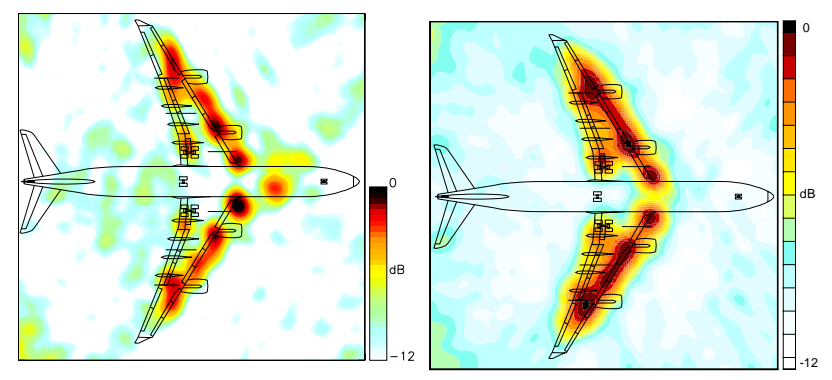

Fig. 6 Slat-and-flap configuration. Comparison of HF (left) and VHF (right) array maps for a high frequency, $\theta_{e}=90^{\circ}$. The $\mathbf{H F}$ array has a slightly better resolution but is more disturbed by side lobes, such as on the fuselage.

"strength" of each landing gear source is to integrate over each area : this might be achieved by reducing the array size until the beam-width is wide enough and the maximal value is stable.

The aircraft altitude has a similar effect. A doubling in the array-source distance induces a doubling in the array spatial resolution. For the case of distributed sources, which is the actual case, this yields higher sound-pressure levels in the maps obtained for higher flyovers.

Figs. 5 and 6 also illustrate this property of arrays with different sizes, for a slat-and-flap configuration. However, in this situation where sources are much more extended compared to the very "localized" areas in the landing-gear case, the level derivation from the map is not possible.

The spatial resolution can also be influenced by the shading of the microphone signals (factors $g_{i}$ in Eq. (7)). An example is shown in Fig. 7. A uniform shading was used in the map on the left side while the weighting of the inner microphones was reduced 


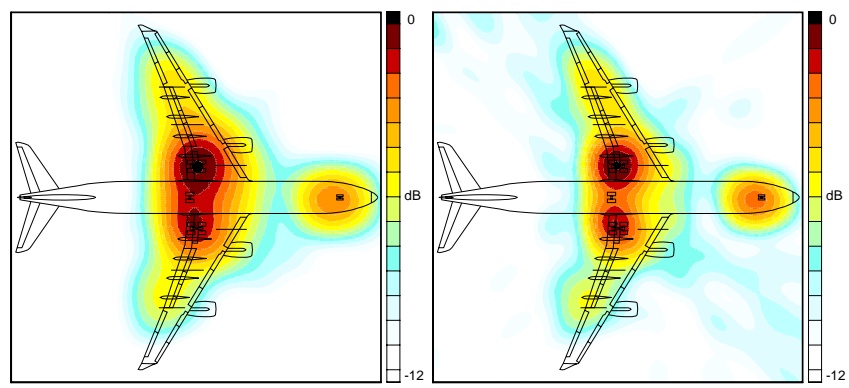

Fig. 7 Comparison of VHF array maps in a veryhigh frequency band, obtained with two different shadings in the case of a landing configuration $\left(\theta_{e}=\right.$ $\left.90^{\circ}\right)$.

in the map on the right side. The improved spatial resolution on the right side is offset by a decrease of dynamic range of the analysis.

\section{Angle of observation and source directivity}

Figure 9 presents the maps in the three directions of investigation for a flap-extended only configuration. All three maps are normalized to the same maximum value, to allow a "source level" comparison. The higher levels noticed at $60^{\circ}$ (forward arc of emission), with the conventions of normalization applied (and assuming that all spurious effects are corrected), would indicate that at this frequency the sound level perceived on the ground is higher in the forward arc. While flap only is extended, the engine inlets do contribute substantially to the overall level. Sources in the rear arc of emission are emphasized behind the engines. The interpretation is here more difficult when it comes to separate the influence of engine and flap noise : the spatial resolution is lower in this direction (as mentioned in Fig. 1, the Doppler effect induces changes in the spatial resolution). The wake behind the wing, the gears and the engines modify the rear arc sound propagation, possibly inducing higher diffusion effects.

The flap sources, in this case appear directive to the forward arc. However, this finding should be cross-checked with the averaged de-Dopplerized spectra, because of the inherent uncertainties of the levels in localization maps.

\section{Example of a map at high frequency}

An example of a map at $8 \mathrm{kHz}$ with the VHF array is shown in Fig. 8. For very high frequencies, the dynamic range is reduced so that the main sources only can be identified. Moreover, the other factors mentioned also affect the absolute levels and even map comparisons between different angles or flyovers. The

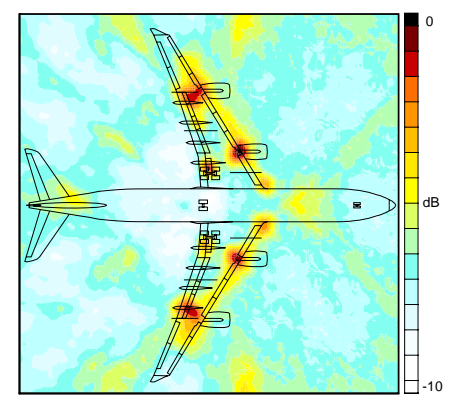

Fig. 8 Localization map obtained with the VHF array at $8 \mathrm{kHz}$. Though the dynamic range is smaller than at lower frequencies, the main sources can still be identified.

main limitation to high-frequency maps is the digitization dynamics on one side, and the propagation attenuation and diffusion on the other side. The digitization of only 16 bits does not allow a proper investigation of the very high frequency components because they are extremely weak compared to lower frequencies. The effects of attenuation due to atmospheric absorption by water vapor, oxygen and nitrogen increase strongly with the frequency. Corrections were not applied here, but should be applied especially if weather conditions are not stable during the flight test campaign. The correction process appears quite tricky here, since this attenuation is both dependent on frequency and distance, therefore depending on the observation angle because of the Doppler effect. Finally, it must be mentioned that no correction is applied for the influences of wind and temperature profiles, since this would first require a precise measurement of them and also a modification of the propagation code used.

\section{Conclusions}

The phased microphone array is shown to be a powerful tool for localizing the sound sources on flying aircraft. A good spatial resolution at low frequencies requires either low flyover altitudes or large arrays. It is demonstrated that the use of large arrays in connection with high flyover altitudes is possible. An advantage of higher altitudes is that the integration time available for a narrow-band analysis is longer. Though, propagation over larger distances imply higher effects of turbulence diffusion, coherence losses on the signals, so that a flyover altitude of about 100 meters is a good compromise.

The study showed that nested arrays must be used for good localization over a large frequency range. The largest array with a size of $16 \mathrm{~m}$ made it possible to separate the sources on the main landing gear down to $500 \mathrm{~Hz}$. A doubling of this size seems to be possible 

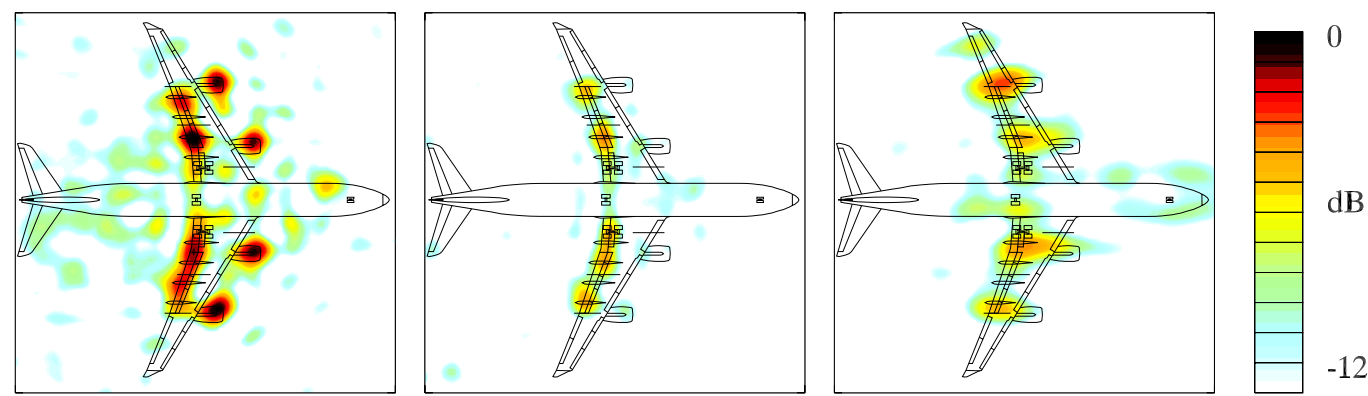

Fig. 9 Localization maps at 60, 90 and $120^{\circ}$ in a one-third octave band in the low frequency domain for a flap-only configuration.

for the analysis of lower frequencies. The smallest array had a sufficient dynamic range to localize the dominating noise sources at $8 \mathrm{kHz}$.

The investigation of the strengths of the noise sources is a much more difficult task than of their locations because the calculated levels for source distributions depend on many parameters, such as

- array performance related to geometry (angles, distance, array layout and size),

- source characteristics (spatial extent, directivity, coherence within the source region...),

- propagation (attenuation, atmospheric turbulence...),

- the Doppler-shifted frequency and its effects on other factors,

- the signal processing (microphone shading, the interpolation method in the de-Dopplerization process...),

- the microphones (size, distance of microphone membranes from the reflecting surface...)

Hence, qualitative information can be derived from localization maps, and in some specific cases quantitative data can be assessed : for example when comparing sources of identical extension seen with equal array performance, or when they are perfectly spatially separated. A quantitative assessment of noise sources would require a perfect estimation of all effects involved, and furthermore the possible use of a multi-pole source model instead of the monopole one used here.

\section{$\underline{\text { Acknowledgments }}$}

These tests were supported by Airbus Industrie and by the European Commission through the RAIN (Re- duction of Airframe and Installation Noise) Project. The authors wish to thank Hugues Remy, Michel Ballet and Airbus France staff, and the teams that participated in the source localization achievement, especially Jacques Chombart, Patrick Lebigot, Bruno Mazin and Daniel Zuba from ONERA and Weiyang Qiao, Joachim Helbig, Konrad Krawczyk, Horst Mettchen (from DLR) and Albrecht Ebner (from TU Berlin).

\section{References}

${ }^{1}$ Michel, U., Barsikow, B., Helbig, J., Hellmig, M., and Schüttpelz, M., "Flyover noise measurements on landing aircraft with a microphone array," AIAA Paper 98-2336, 1998, 4th AIAA/CEAS Aeroacoustics Conference, Toulouse, France, June $2-4,1998$.

${ }^{2}$ Piet, J.-F., Elias, G., and Lebigot, P., "Localization of acoustic sources from a landing aircraft with a microphone array," AIAA Paper 99-1811, 1999, 5th AIAA/CEAS Aeroacoustics Conference, Bellevue, Washington, USA, May 10-12, 1999.

${ }^{3}$ Michel, U. and Qiao, W., "Directivity of landing gear noise based on flyover measurements," AIAA Paper 99-1956, 1999, 5th AIAA/CEAS Aeroacoustics Conference, Bellevue, Wa, May $10-12,1999$.

${ }^{4}$ Chow, L. C., Mau, K., and Remy, H., "Landing Gear and High Lift Devices Airframe Noise Research," AIAA Paper 2002-2408, 2002, 8th AIAA/CEAS Aeroacoustics Conference, Breckenridge, Co, June 17-19, 2002.

${ }^{5}$ Davy, R., Moens, F., and Remy, H., "Aeroacoustic Behaviour of a 1:11 Scale Airbus Model in the Open Anechoic Wind Tunnel CEPRA 19," AIAA Paper 2002-2412, 2002, 8th AIAA/CEAS Aeroacoustics Conference, Breckenridge, Co, June $17-19,2002$.

${ }^{6}$ Howell, G. P., Bradley, A. J., McCormick, M. A., and Brown, J. D., "De-Dopplerization and acoustic imaging of aircraft flyover noise measurements," J. Sound Vib., Vol. 105, 1986, pp. 151-167.

${ }^{7}$ Elias, G., "Source localization with a two-dimensional focussed array: optimal signal processing for a cross-shaped ar- 
ray," 1995, Inter-Noise 95, Newport Beach (USA), July 10-12, 1995.

${ }^{8}$ Piet, J.-F. and Elias, G., "Airframe noise source localization using a microphone array," AIAA Paper 97-1643, 1997, 3rd AIAA/CEAS Aeroacoustics Conference, Atlanta, Ga, May 1214, 1997.

${ }^{9}$ Sen, R., "Interpretation of Acoustic Source Maps made with an Elliptic-Mirror Directional Microphone System," AIAA Paper 96-1712, 1996, 2nd AIAA/CEAS Aeroacoustics Conference, State College, Pa, May 6-8, 1996.

${ }^{10}$ Mosher, M., "Phased Arrays for Aerocoustic Testing: Theoretical Development," AIAA Paper 96-1713, 1996, 2nd AIAA/CEAS Aeroacoustics Conference, State College, Pa, May 6-8, 1996.

${ }^{11}$ Varnier, J., Piet, J., Gely, D., Elias, G., and Radulovic, S., "Modelling of The Acoustic Environment on the Ariane 5 Fairing Using Small Scale Test Data," AIAA Paper 80-0973, 1980, 2nd AIAA/CEAS Aeroacoustics Conference, State College, Pa, May, 1996. 